\title{
[3]Ferrocenophanes with a Tetramethyldisiloxane Bridge: Synthesis and Molecular Structure
}

\author{
U. Siemeling*, R. Krallmann, P. Jutzi, B. Neumann, and H.-G. Stammler \\ Fakultät für Chemie, Universität Bielefeld, D-33615 Bielefeld, Federal Republic of Germany
}

\begin{abstract}
Summary. 6,6,8,8-Tetramethyl-7-oxa-6,8-disila[3]ferrocenophane 2 was obtained from the di(alkoxysilyl) ferrocene $\left(\mathrm{H}_{4} \mathrm{C}_{5} \mathrm{SiMe}_{2} \mathrm{OR}\right)_{2} \mathrm{Fe}\left(\mathrm{R}=\mathrm{CH}_{2} \mathrm{CH}_{2} \mathrm{OCH}_{2} \mathrm{CH}_{2} \mathrm{OCH}_{2} \mathrm{CH}_{2} \mathrm{OMe}\right)$ by hydrolysis and subsequent intramolecular disiloxane formation. $2,2^{\prime}, 3,3^{\prime}, 4,4^{\prime}, 5,5^{\prime}, 6,6,8,8$-Dodecamethyl-7-oxa-6,8disila-[3]ferrocenophane 3 was formed by air oxidation of $2,2^{\prime} 3,3^{\prime}, 4,4^{\prime}, 5,5^{\prime}, 6,6,7,7$-dodecamethyl-6,7disila[2]ferrocenophane. The crystal structures of both compounds were determined by single-crystal $X$-ray diffraction (2: $a=8.5330(10), b=15.610(3), c=18.774(5) \AA, \alpha=70.68(2), \beta=77.94(2), \gamma=$ $75.150(10)^{\circ}, V=2259.8(8) \AA^{3}, Z=6$, space group $\mathrm{P} \overline{1}, \quad R=0.045, R_{w}=0.044 ; 3: a=12.388(3)$, $b=9.924(3), c=19.136(10) \AA, \beta=105.11(3)^{\circ}, V=2271.2(15) \AA^{3}, Z=4$, space group $\mathrm{P} 2_{1} / \mathrm{c}, R=0.076$, $R_{w}=0.060$ ). Owing to the flexibility of the disiloxane bridge, 2 and 3 are unstrained molecules.
\end{abstract}

Keywords. Ferrocenophane; Disiloxane unit.

\section{[3]Ferrocenophane mit Tetramethyldisiloxan-Brücke: Synthese und Molekülstruktur}

Zusammenfassung. 6,6,8,8-Tetramethyl-7-oxa-6,8-disila[3]-ferrocenophan 2 entsteht aus dem Di(alkoxysilyl)ferrocen $\left(\mathrm{H}_{4} \mathrm{C}_{5} \mathrm{SiMe}_{2} \mathrm{OR}\right)_{2} \mathrm{Fe}\left(\mathrm{R}=\mathrm{CH}_{2} \mathrm{CH}_{2} \mathrm{OCH}_{2} \mathrm{CH}_{2} \mathrm{OCH}_{2} \mathrm{CH}_{2} \mathrm{OMe}\right)$ durch Hydrolyse und anschließende intramolekulare Disiloxan-Bildung. 2,2',3,3',4,4',5,5',6,6,8,8-Dodecamethyl-7-oxa-6,8-disila[3]ferrocenophan 3 wurde durch Luftoxidation von 2, $2^{\prime}, 3,3^{\prime}, 4,4^{\prime}, 5,5^{\prime}, 6,6,7,7$ Dodecamethyl-6,7-disila[2]ferrocenophan erhaeten. Die Kristallstrukturen beider Verbindungen wurden durch Einkristall-Röntgenstrukturanalyse bestimmt (2: $a=8.5330(10), b=15.610(3)$, $c=18.774(5) \AA, \alpha=70.68(2), \beta=77.94(2), \gamma=75.150(10)^{\circ}, V=2259.8(8) \AA^{3}, Z=6$, Raumgruppe $\mathrm{P} \overline{1}$, $R=0.045, R_{w}=0.044 ; 3: a=12.388(3), b=9.924(3), c=19.136(10) \AA, \beta=105.11(3)^{\circ}, V=2271.2(15) \AA^{3}$, $Z=4$, Raumgruppe $\mathrm{P} 2_{1} / \mathrm{c}, R=0.076, R_{w}=0.060$ ). Wegen der Flexibilität der Disiloxan-Brücke sind 2 und 3 ungespannte Moleküle.

\section{Introduction}

Ferrocenophanes represent a fascinating class of compounds. They have been extensively investigated over the past 35 years. The research on "all-carbon-bridged" species has culminated in the synthesis of "superferrocenophane" by Hisatome et al. [1]. Currently, heteroatom-bridged ferrocenophanes are receiving much attention due to special electronic and steric features of the bridge(s) [2]. Recently, we described the synthesis and structure of the [3] [3]ferrocenophane 1, which is the 
first example of a ferrocenophane bearing two disiloxane bridges; it is also the first example of a structurally characterized disiloxane-bridged ferrocenophane [3].

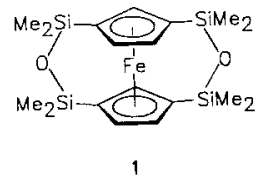

Compound 1 was formed from the tetra(alkoxysilyl)ferrocene $\left[\mathrm{H}_{3} \mathrm{C}_{5}\left(\mathrm{SiMe}_{2}\right.\right.$ $\left.\mathrm{OR})_{2}\right]_{2} \mathrm{Fe}\left(\mathrm{R}=\mathrm{CH}_{2} \mathrm{CH}_{2} \mathrm{OCH}_{2} \mathrm{CH}_{2} \mathrm{OMe}\right)$ [3] by hydrolysis and subsequent intramolecular disiloxane formation; diethylene glycol monomethyl ether was identified as the second reaction product.

We now wish to report the synthesis and structure of two closely related species, namely the disiloxane-bridged [3]ferrocenophanes $\mathbf{2}$ and $\mathbf{3}$.

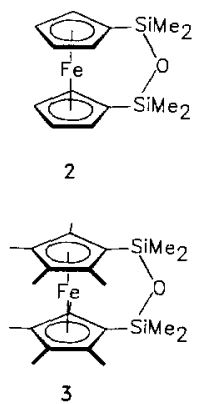

\section{Results and Discussion}

Compound 2 [4] is formed in analogy to 1 from the di(alkoxysilyl)ferrocene $\left(\mathrm{H}_{4} \mathrm{C}_{5} \mathrm{SiMe}_{2} \mathrm{OR}\right)_{2} \mathrm{Fe}\left(\mathrm{R}=\mathrm{CH}_{2} \mathrm{CH}_{2} \mathrm{OCH}_{2} \mathrm{CH}_{2} \mathrm{OCH}_{2} \mathrm{CH}_{2} \mathrm{OMe}\right)$ upon prolonged standing in the air at room temperature; triethyleneglycol monomethyl ether is the second reaction product, as identified by ${ }^{1} \mathrm{H}-\mathrm{NMR}$ spectroscopy.

An alternative strategy for the formation of a disiloxane-bridged species involves the insertion of oxygen into a somewhat strained disilanediyl-bridged compound [5]. Indeed, when an acetonitrile solution of the disilanediyl-bridged [2]ferrocenophane $\left(\mathrm{Me}_{4} \mathrm{C}_{5} \mathrm{SiMe}_{2}\right)_{2} \mathrm{Fe}$ [6] was kept in the air at room temperature for several days, crystals of the disiloxane-bridged species 3 precipitated [7].

The molecular structures of $\mathbf{2}$ and $\mathbf{3}$ (Figs. 1 and 2) were established from singlecrystal X-ray diffraction data. Atomic coordinates and thermal parameters for 2 and 3 are presented in Tables 1 and 2.

For compound 2, the asymmetric unit of the unit cell contains three crystallographically independent molecules, two of which are very similar, but not identical within the margin of error of the structure determination; the third molecule differs from the other two mainly by the significantly higher $\mathrm{Cp}$-ring twist angle $(7.0 \mathrm{vs}$. ca. $\left.1^{\circ}\right) .2$ and 3 are virtually unstrained: for both compounds, the cyclopentadienyl rings adopt an eclipsed conformation; ring twist angles range from 1.1 to $7.0^{\circ}$. The cyclo- 

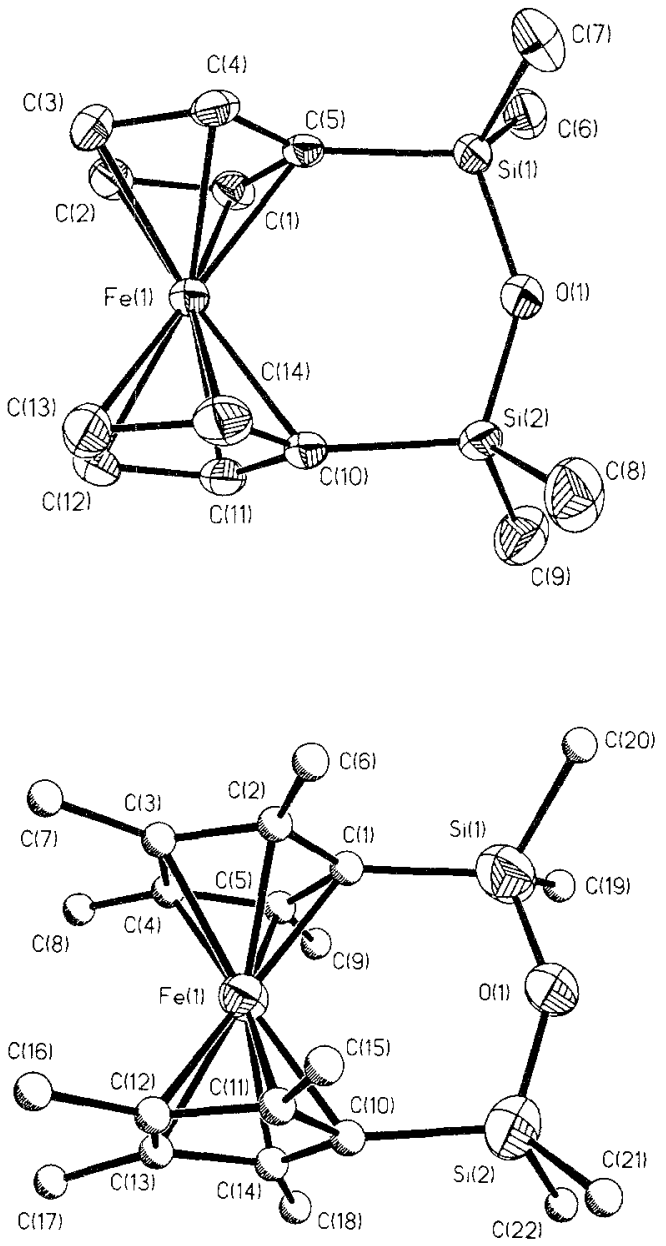

Fig. 1. Molecular structure of compound 2 (molecule 1 of three crystallographically independent molecules). Selected bond lengths $(\AA)$ and angles $\left({ }^{\circ}\right): \mathrm{C}(1)-\mathrm{C}(2) \quad 1.422(5), \quad \mathrm{C}(1)-\mathrm{C}(5) \quad 1.444(5)$, $\mathrm{C}(2)-\mathrm{C}(3) 1.419(5), \mathrm{C}(3)-\mathrm{C}(4) 1.422(5), \mathrm{C}(4)-\mathrm{C}(5)$ 1.429(5), $\mathrm{C}(10)-\mathrm{C}(11) 1.442(5), \mathrm{C}(10)-\mathrm{C}(14) 1.434(5)$, $\mathrm{C}(11)-\mathrm{C}(12)$ 1.422(6), $\mathrm{C}(12)-\mathrm{C}(13) 1.412(6), \mathrm{C}(13)-$ $\mathrm{C}(14) \quad 1.437(6), \mathrm{Si}(1)-\mathrm{C}(5) \quad 1.861(4), \mathrm{Si}(1)-\mathrm{O}(1)$ 1.637(3), $\mathrm{Si}(2)-\mathrm{C}(10)$ 1.859(4), $\mathrm{Si}(2)-\mathrm{O}(1) 1.630(4)$; $\mathrm{O}(1)-\mathrm{Si}(1)-\mathrm{C}(5)$ 110.3(2), Si(1)-O(1)-Si(2) 143.6(2), $\mathrm{O}(1)-\mathrm{Si}(2)-\mathrm{C}(10)$ 111.1(2)

Fig. 2. Molecular structure of compound 3. Selected bond lengths $(\AA)$ and angles $\left(^{\circ}\right)$ (the cyclopentadienyl units were refined as regular pentagons with $\mathrm{C}-\mathrm{C}=1.420 \AA)$ : $\mathrm{Si}(1)-\mathrm{C}(1) 1.896(14), \mathrm{Si}(1)-$ $\mathrm{O}(1) 1.618(17), \mathrm{Si}(2)-\mathrm{C}(10)$ 1.886(14), $\mathrm{Si}(2)-\mathrm{O}(1)$ 1.623(18); $\mathrm{O}(1)-\mathrm{Si}(1)-\mathrm{C}(1) 110.2(9), \mathrm{Si}(1)-\mathrm{O}(1)-$ $\mathrm{Si}(2)$ 139.7(9), O(1)-Si(2)-C(10) 110.4(7)

pentadienyl rings are very nearly parallel; ring tilt angles are close to zero $\left(0.7\right.$ to $\left.2.7^{\circ}\right)$. The iron-ring centroid distance is $1.65 \AA$ for both compounds, which is almost identical to the values observed for ferrocene and decamethyl ferrocene (1.66 $\AA$ ) [8]. $\mathrm{The} \mathrm{Si}-\mathrm{O}-\mathrm{Si}$ angles range from 139.7 to $143.6^{\circ}$, which is at the low end of the region typical of disiloxanes [9].

It is instructive to compare the molecular structure of the disiloxane-bridged [3]ferrocenophanes $\mathbf{2}$ and $\mathbf{3}$ with that of [3]ferrocenophane $\mathbf{4}$ and also with that of 7-oxa[3]ferrocenophane 5 (Fig. 3). Pertinent data are collected in Table 3.

It is obvious that compounds $\mathbf{4}$ and $\mathbf{5}$ are fairly strained: Their iron-ring centroid distance is $0.02 \AA$ shorter than that found for $\mathbf{2}$ and $\mathbf{3}$; furthermore, a considerable ring tilt is observed (7.6 and $11.9^{\circ}$, respectively). Additionally, the angle $\alpha$ (see Fig. 3), which also gives a rough measure of the molecular strain, deviates slightly more from $180^{\circ}$ for compounds $\mathbf{4}$ and $\mathbf{5}$ than it does for $\mathbf{2}$ and $\mathbf{3}$. The main factor for the unstrained character of $\mathbf{2}$ and $\mathbf{3}$ is the central bridge angle $\beta$, which is ca. $140^{\circ}$ for $\mathbf{2}$ and 3 , but only $123^{\circ}$ for $\mathbf{4}$ and a mere $114.7^{\circ}$ for $\mathbf{5}$. This reflects the remarkable flexibility of disiloxane units, which can easily adjust to a wide range of steric requirements [10]. 
Table 1. Atomic coordinates $\left(\times 10^{4}\right)$ and equivalent isotropic displacement coefficients $\left(\AA^{2} \times 10^{3}\right)$ for compound 2

\begin{tabular}{|c|c|c|c|c|}
\hline & $\mathbf{x}$ & $\mathrm{y}$ & $\mathrm{z}$ & $U(\mathrm{eq})$ \\
\hline $\mathrm{Fe}(1)$ & $1959(1)$ & $7213(1)$ & $5045(1)$ & $21(1)$ \\
\hline $\mathrm{Fe}(2)$ & $1366(1)$ & $7871(1)$ & $1522(1)$ & $19(1)$ \\
\hline $\mathrm{Fe}(3)$ & $4180(1)$ & $7609(1)$ & $8515(1)$ & $20(1)$ \\
\hline Si(1) & $-1540(1)$ & $6798(1)$ & $4681(1)$ & $24(1)$ \\
\hline $\mathrm{Si}(2)$ & $-1774(1)$ & $8731(1)$ & $4868(1)$ & $26(1)$ \\
\hline $\mathrm{Si}(3)$ & $5061(1)$ & $6359(1)$ & $1872(1)$ & $23(1)$ \\
\hline $\mathrm{Si}(4)$ & $4795(1)$ & $8379(1)$ & $1862(1)$ & $25(1)$ \\
\hline $\operatorname{si}(5)$ & $7896(1)$ & $8251(1)$ & $8258(1)$ & $22(1)$ \\
\hline $\mathrm{Si}(6)$ & $8029(1)$ & $6254(1)$ & $8269(1)$ & $23(1)$ \\
\hline$O(1)$ & $-2210(3)$ & $7881(2)$ & $4676(2)$ & $45(1)$ \\
\hline$O(2)$ & $5407(3)$ & $7253(2)$ & $2045(2)$ & $36(1)$ \\
\hline$o(3)$ & $8561(3)$ & $7248(2)$ & $8088(2)$ & $37(1)$ \\
\hline$C(1)$ & $2040(4)$ & $6655(2)$ & $4192(2)$ & $23(1)$ \\
\hline$C(2)$ & $3515(4)$ & $6290(3)$ & $4524(2)$ & $27(2)$ \\
\hline$G(3)$ & $3105(4)$ & $5837(2)$ & $5309(2)$ & $27(1)$ \\
\hline$C(4)$ & $1376(4)$ & $5926(2)$ & $5459(2)$ & $23(1)$ \\
\hline$C(5)$ & $679(4)$ & $6437(2)$ & $4774(2)$ & $20(1)$ \\
\hline$C(6)$ & $-1848(5)$ & $6721(3)$ & $3755(2)$ & $34(2)$ \\
\hline$c(7)$ & $-2697(5)$ & $6062(3)$ & $5490(2)$ & $36(2)$ \\
\hline$C(8)$ & $-3255(5)$ & $8959(3)$ & $5688(2)$ & $45(2)$ \\
\hline$c(9)$ & $-1975(5)$ & $9737(3)$ & $4012(2)$ & $44(2)$ \\
\hline$C(10)$ & $343(4)$ & $8428(2)$ & $5111(2)$ & $26(1)$ \\
\hline$C(11)$ & $1794(4)$ & $8618(3)$ & $4586(2)$ & $29(2)$ \\
\hline$C(12)$ & $3192(5)$ & $8214(3)$ & $4973(3)$ & $36(2)$ \\
\hline$c(13)$ & $2646(5)$ & $7775(3)$ & $5740(3)$ & $37(2)$ \\
\hline$C(14)$ & $894(5)$ & $7910(3)$ & $5833(2)$ & $32(2)$ \\
\hline$C(15)$ & $1473(4)$ & $6535(2)$ & $2194(2)$ & $23(1)$ \\
\hline$C(16)$ & $88(5)$ & $6836(2)$ & $1799(2)$ & $27(1)$ \\
\hline$C(17)$ & $660(5)$ & $7082(3)$ & $1005(2)$ & $30(2)$ \\
\hline$C(18)$ & $2411(5)$ & $6944(3)$ & $910(2)$ & $27(1)$ \\
\hline$C(19)$ & $2938(4)$ & $6599(2)$ & $1657(2)$ & $21(1)$ \\
\hline$C(20)$ & $6511(5)$ & $6131(3)$ & $1039(2)$ & $38(2)$ \\
\hline$C(21)$ & $5383(5)$ & $5367(3)$ & $2733(2)$ & $39(2)$ \\
\hline$c(22)$ & $6112(5)$ & $8954(3)$ & $1012(2)$ & $41(2)$ \\
\hline$C(23)$ & $4946(5)$ & $8672(3)$ & $2724(2)$ & $33(2)$ \\
\hline$c(24)$ & $2637(4)$ & $8745(2)$ & $1668(2)$ & $22(1)$ \\
\hline$C(25)$ & $2079(4)$ & $9103(2)$ & $931(2)$ & $26(1)$ \\
\hline$c(26)$ & $339(5)$ & $9225(3)$ & $1036(2)$ & $31(2)$ \\
\hline$c(27)$ & $-215(4)$ & $8962(3)$ & $1829(2)$ & $30(2)$ \\
\hline$C(28)$ & $1181(4)$ & $8672(2)$ & $2220(2)$ & $26(1)$ \\
\hline$C(29)$ & $4539(4)$ & $8884(2)$ & $7841(2)$ & $26(1)$ \\
\hline$c(30)$ & $2894(4)$ & $8949(2)$ & $8203(2)$ & $27(1)$ \\
\hline$C(31)$ & $2912(4)$ & $8632(3)$ & $9005(2)$ & $28(2)$ \\
\hline$c(32)$ & $4595(4)$ & $8381(2)$ & $9129(2)$ & $24(1)$ \\
\hline$C(33)$ & $5617(4)$ & $8534(2)$ & $8409(2)$ & $20(1)$ \\
\hline$C(34)$ & $8714(5)$ & $9122(3)$ & $7418(2)$ & $34(2)$ \\
\hline$C(35)$ & $8652(5)$ & $8200(3)$ & $9130(2)$ & $35(2)$ \\
\hline$C(36)$ & $8808(5)$ & $5438(3)$ & $9145(2)$ & $41(2)$ \\
\hline$C(37)$ & $8968(5)$ & $5836(3)$ & $7431(2)$ & $42(2)$ \\
\hline$C(38)$ & $5772(4)$ & $6407(2)$ & $8396(2)$ & $26(1)$ \\
\hline$C(39)$ & $4689(5)$ & $6227(3)$ & $9104(2)$ & $31(2)$ \\
\hline$C(40)$ & $3044(5)$ & $6496(3)$ & $8952(3)$ & $38(2)$ \\
\hline$C(41)$ & $3042(5)$ & $6847(3)$ & $8154(3)$ & $37(2)$ \\
\hline$c(42)$ & $4711(5)$ & $6798(3)$ & $7803(2)$ & $32(2)$ \\
\hline
\end{tabular}

* Equivalent isotropic $U$ defined as one third of the trace of the orthogonalized $U_{\mathrm{ij}}$ tensor

\section{Experimental Part}

NMR: Bruker AM $300\left(300.133 \mathrm{MHz},{ }^{1} \mathrm{H}\right.$, ext. TMS). EI-MS: Varian CH5 and VG Autospec (70 eV).

2: $1.20 \mathrm{~g}(1.91 \mathrm{mmol})$ of the di(alkoxysilyl)ferrocene $\left(\mathrm{H}_{4} \mathrm{C}_{5} \mathrm{SiMe}_{2} \mathrm{OR}\right)_{2} \mathrm{Fe}\left(\mathrm{R}=\mathrm{CH}_{2} \mathrm{CH}_{2} \mathrm{OCH}_{2} \mathrm{CH}_{2}\right.$ $\mathrm{OCH}_{2} \mathrm{CH}_{2} \mathrm{OMe}$ ) [3] are stored in the air at room temperature for several months. The oil is removed 
Table 2. Atomic coordinates $\left(\times 10^{4}\right)$ and equivalent isotropic displacement coefficients $\left(\AA^{2} \times 10^{3}\right)$ for compound 3

\begin{tabular}{|c|c|c|c|c|}
\hline & $\mathrm{x}$ & $\mathrm{y}$ & $\mathbf{Z}$ & $U(\mathrm{eq})$ \\
\hline $\mathrm{Fe}(1)$ & $2432(3)$ & $1025(3)$ & $2582(2)$ & $34(1)$ \\
\hline $\operatorname{Si}(1)$ & $3264(7)$ & $492(8)$ & $1019(4)$ & $58(4)$ \\
\hline Si(2) & $1512(7)$ & $2671(9)$ & $950(4)$ & $63(4)$ \\
\hline $0(1)$ & $2193(13)$ & $1449(16)$ & $696(7)$ & $55(8)$ \\
\hline$C(1)$ & $3410(13)$ & $142(17)$ & $2013(6)$ & $36(7)$ \\
\hline$C(2)$ & 2769 & -847 & 2261 & $45(7)$ \\
\hline$C(3)$ & 3049 & -786 & 3029 & $55(8)$ \\
\hline $\mathrm{C}(4)$ & 3864 & 241 & 3255 & $40(7)$ \\
\hline$C(5)$ & 4087 & 814 & 2628 & $35(7)$ \\
\hline$C(6)$ & $1948(19)$ & $-1826(24)$ & $1814(12)$ & $85(9)$ \\
\hline$c(7)$ & $2625(22)$ & $-1687(27)$ & $3524(13)$ & $135(14)$ \\
\hline$c(8)$ & $4455(19)$ & $552(26)$ & $4019(11)$ & $107(11)$ \\
\hline$c(9)$ & $4976(22)$ & $1841(24)$ & $2628(13)$ & $91(10)$ \\
\hline$C(10)$ & $1451(11)$ & 2411 (13) & $1915(6)$ & $25(6)$ \\
\hline$C(11)$ & 760 & 1427 & 2120 & $46(7)$ \\
\hline $\mathrm{C}(12)$ & 992 & 1431 & 2887 & $42(7)$ \\
\hline$c(13)$ & 1826 & 2418 & 3156 & $43(7)$ \\
\hline$c(14)$ & 2110 & 3024 & 2556 & $45(8)$ \\
\hline$c(15)$ & -108 & 515 & 1628 & $77(9)$ \\
\hline$c(16)$ & $439(19)$ & $576(25)$ & $3347(12)$ & $97(10)$ \\
\hline$C(17)$ & $2247(18)$ & $2865(24)$ & $3969(10)$ & $79(9)$ \\
\hline$C(18)$ & $2845(17)$ & $4183(22)$ & $2640(11)$ & $78(9)$ \\
\hline$C(19)$ & $4479(21)$ & 1381 (28) & $861(12)$ & $121(12)$ \\
\hline$c(20)$ & $3089(22)$ & $-989(30)$ & $446(13)$ & $160(13)$ \\
\hline$C(21)$ & $131(20)$ & $2741(24)$ & $296(11)$ & $96(10)$ \\
\hline$C(22)$ & $2205(19)$ & $4298(25)$ & $833(12)$ & $97(11)$ \\
\hline
\end{tabular}

* Equivalent isotropic $U$ defined as one third of the trace of the orthogonalized $U_{\mathrm{ij}}$ tensor

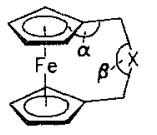

$4\left(x=\mathrm{CH}_{2}\right)$

$5(x=0)$

Fig. 3. Compounds 4 and 5. The angles $\alpha$ and $\beta$ give an indication of the molecular strain in these compounds ( $\alpha$ is the angle between the vector defined by the bridge atom and its $\mathrm{Cp}$ carbon neighbour and the plane defined by this carbon atom and its two $\mathrm{Cp}$ carbon neighbours; $\beta$ is the central bridge angle at $\mathrm{X}$ )

Table 3. Compilation of parameters indicative of molecular strain for compounds 2-5

\begin{tabular}{|c|c|c|c|c|c|c|}
\hline Compound & & $2^{\mathrm{a}}$ & & 3 & $4^{\mathrm{b}}$ & $5^{\mathrm{b}}$ \\
\hline Iron-ring centroid distance $(\AA)$ & & 1.65 & & 1.65 & 1.63 & 1.63 \\
\hline $\mathrm{Cp}$ ring tilt $\left(^{\circ}\right)$ & 0.7 & 0.8 & 2.7 & 1.5 & 7.6 & 11.9 \\
\hline $\mathrm{Cp}$ ring twist $\left(^{\circ}\right)$ & 7.0 & 1.1 & 1.2 & 3.3 & 0.9 & 1.4 \\
\hline $180-\alpha\left({ }^{\circ}\right)$ & $2.6 / 3.1$ & $3.1 / 2.7$ & $3.0 / 3.3$ & $2.6 / 4.1$ & $4.5 / 6.4$ & $5.2 / 4.3$ \\
\hline$\beta\left(^{\circ}\right)$ & 143.6 & 141.6 & 140.9 & 139.7 & 123.0 & 114.7 \\
\hline
\end{tabular}

a Three crystallographically independent molecules

b Data taken from: Hillman M., Austin J. D. (1987) Organometallics 6: 1737

from the large orange crystals by using filter paper. Yield $575 \mathrm{mg}(95 \%)$. ${ }^{1} \mathrm{H}-\mathrm{NMR}\left(\mathrm{CDCl}_{3}\right): \delta$ $(\mathrm{ppm})=0.27(\mathrm{~s}, 12 \mathrm{H}, \mathrm{Me}), 4.22(\mathrm{~s}, 4 \mathrm{H}, \mathrm{CH}), 4.31(\mathrm{~s}, 4 \mathrm{H}, \mathrm{CH}) . \mathrm{MS}: \mathrm{m} / \mathrm{z}(\%)=316(100)\left[\mathrm{M}^{+}\right]$.

3: A solution of $45 \mathrm{mg}(0.11 \mathrm{mmol})$ of 2,2',3,3,4,4,5,5',6,6,7,7-dodecamethyl-6,7-disila[2]ferrocenophane [6] in $15 \mathrm{ml}$ of acetonitrile is allowed to stand in the air at room temperature for several days. The 
Table 4. Single-crystal X-ray diffraction study of compounds 2 and 3: Crystal data

\begin{tabular}{lll}
\hline Compound & $\mathbf{2}$ & $\mathbf{3}$ \\
\hline Empirical formula & $\mathrm{C}_{14} \mathrm{H}_{20} \mathrm{FeOSi}_{2}$ & $\mathrm{C}_{22} \mathrm{H}_{36} \mathrm{FeOSi}_{2}$ \\
orange & & $\begin{array}{l}\text { orange } \\
\text { Colour }\end{array}$ \\
Crystal size (mm) & $0.5 \times 0.7 \times 0.7$ & monoclinic \\
Crystal system & triclinic & $\mathrm{P} 2{ }_{1} / \mathrm{c}$ \\
Space group & $\mathrm{P} \overline{1}$ & $a=12.388(3) \AA$ \\
Unit cell dimensions & $a=8.5330(10) \AA$ & $b=9.924(3) \AA$ \\
& $b=15.610(3) \AA$ & $c=19.136(10) \AA$ \\
& $c=18.774(5) \AA$ & \\
& $\alpha=70.68(2)^{\circ}$ & $\beta=105.11(3)^{\circ}$ \\
& $\beta=77.94(2)^{\circ}$ & \\
Volume & $\gamma=75.150(10)^{\circ}$ & $2271.2(15) \AA^{3}$ \\
$Z$ & $2259.8(8) \AA^{3}$ & 4 \\
Formula weight & 6 & 428.5 \\
Density (calcd.) & 316.3 & $1.253 \mathrm{~g} / \mathrm{cm}^{3}$ \\
Absorption coefficient $(\mu)$ & $1.395 \mathrm{~g} / \mathrm{cm}^{3}$ & $0.774 \mathrm{~mm}^{-1}$ \\
F(000) & 996 & 920 \\
\hline
\end{tabular}

Table 5. Single-crystal X-ray diffraction study of compounds 2 and 3: Data collection

\begin{tabular}{llc}
\hline Compound & 2 & 3 \\
\hline Radiation & MoK $\alpha(\lambda=0.71073 \AA)$ \\
Temperature $(\mathrm{K})$ & 173 & 294 \\
Monochromator & highly oriented graphite crystal \\
$2 \theta$ range & 3.0 to $55.0^{\circ}$ & 3.0 to $40.0^{\circ}$ \\
Scan type & & $\omega$ \\
Scan speed & variable; 6.0 to & variable; 5.0 to \\
& $29.3^{\circ} /$ min in $\omega$ & $30.0^{\circ} /$ min in $\omega$ \\
Scan range $(\omega)$ & $1.40^{\circ}$ & $1.20^{\circ}$ \\
Background measurement & stationary crystal and stationary \\
& counter at beginning and end of \\
& scan, each for $25.0^{\circ} \%$ of total scan time \\
Standard reflexions & 4 measured every & 3 measured every \\
& 76 reflections & 50 reflections \\
Index ranges & $0 \leqslant h \leqslant 11$ & $0 \leqslant h \leqslant 11$ \\
& $-19 \leqslant k \leqslant 20$ & $0 \leqslant k \leqslant 9$ \\
Reflections collected & $-23 \leqslant l \leqslant 24$ & $-18 \leqslant l \leqslant 17$ \\
Independent reflections & 11127 & 2427 \\
Observed reflections & 10427 & 2137 \\
(F $>5.0 \sigma(F))$ & 7631 & 730 \\
& & \\
\hline
\end{tabular}


Table 6. Single-crystal X-ray diffraction study of compounds 2 and $\mathbf{3}$ :

Structure solution and refinement

\begin{tabular}{|c|c|c|}
\hline Compound & 2 & 3 \\
\hline Programme package used & \multicolumn{2}{|c|}{ Siemens SHELXTL PLUS (VMS) } \\
\hline Solution & \multicolumn{2}{|c|}{ direct method } \\
\hline Refinement method & \multicolumn{2}{|c|}{ full-matrix least-squares } \\
\hline Quantity minimized & \multicolumn{2}{|c|}{$\Sigma \mathrm{w}\left(\mathrm{F}_{0}-\mathrm{F}_{\mathrm{c}}\right)^{2}$} \\
\hline Hydrogen atoms & \multicolumn{2}{|c|}{ riding model, fixed isotropic $U$} \\
\hline Weighting scheme & \multicolumn{2}{|c|}{$\mathrm{w}^{-1}=\sigma^{2}(\mathrm{~F})$} \\
\hline Number of parameters refined & 487 & 104 \\
\hline \multirow[t]{3}{*}{ Final $R$ indices (obs. data) } & $R=0.045$ & $R=0.076$ \\
\hline & $R_{w}=0.044$ & $R_{w}=0.060$ \\
\hline & \multicolumn{2}{|c|}{$R_{w}=\left(\Sigma \mathrm{w}\left(\left|\mathrm{F}_{0}\right|-\left|\mathrm{F}_{\mathrm{c}}\right|\right)^{2} / w\left|\mathrm{~F}_{0}\right|^{2}\right)^{1 / 2}$} \\
\hline \multirow[t]{2}{*}{$R$ indices (all data) } & $R=0.065$ & $R=0.201$ \\
\hline & $R_{w}=0.049$ & $R_{w}=0.077$ \\
\hline Goodness-of-fit & 2.24 & 1.74 \\
\hline Largest and mean $\Delta / \sigma$ & $0.003,0.001$ & $0.001,0.000$ \\
\hline Data-to-parameter ratio & $15.7: 1$ & $7.0: 1$ \\
\hline Largest difference peak & $0.63 \mathrm{e} / \AA^{3}$ & $0.54 \mathrm{e} / \AA^{3}$ \\
\hline Largest difference hole & $-0.62 \mathrm{e} / \AA^{3}$ & $-0.38 \mathrm{e} / \AA^{3}$ \\
\hline
\end{tabular}

orange crystals are isolated by filtration and dried in vacuo. Yield $20 \mathrm{mg}(42 \%) \cdot{ }^{1} \mathrm{H}-\mathrm{NMR}\left(\mathrm{C}_{6} \mathrm{D}_{6}\right): \delta$ $(\mathrm{ppm})=0.49\left(\mathrm{~s}, 12 \mathrm{H}, \mathrm{SiMe}_{2}\right), 1.59(\mathrm{~s}, 12 \mathrm{H}, \mathrm{CMe}), 1.81(\mathrm{~s}, 12 \mathrm{H}, \mathrm{CMe}) \mathrm{MS}: \mathrm{m} / \mathrm{z}(\%)=428(100)[\mathrm{M}]^{+}$.

\section{$X$-Ray Crystallography}

The crystal structures of $\mathbf{2}$ and $\mathbf{3}$ were determined using a Syntex $\mathrm{P} 2_{1}$ four circle diffractometer. Details concerning the structure determinations are collected in Tables 4-6 [11]. Atomic scattering factors were taken from standard sources [12].

\section{Acknowledgments}

This work was financed by the Deutsche Forschungsgemeinschaft and the Fonds der Chemischen Industrie. U.S. wishes to express his sincere gratitude to P. J. for his continued support.

\section{References}

[1] Hisatome M., Watanabe J., Kawajiri Y., Yamakawa K. (1990) Organometallics 9:497

[2] See, for example: Herberhold M., Leitner P., Thewalt U. (1990) Z. Naturforsch. 45b: 1503; Herberhold M., Leitner P. (1991) J. Organomet. Chem. 411: 233; Herberhold M., Dörnhöfer C., Scholz A., Jin G.-X.(1992) Phosphorus Sulfur Silicon Relat. Elem. 64: 161; Zanello P., Opromolla G., Casarin M., Herberhold M., Leitner P. (1993) J. Organomet. Chem. 443: 199

[3] Siemeling U., Neumann B., Stammler H.-G. (1993) Chem. Ber. 126: 1311

[4] The synthesis of 2 was reported as early as 1961 by Schaaf et al. using a strategy similar to ours: Schaaf R. L., Kan P. T., Lenk C. T. (1961) J. Org. Chem. 26: 1790

[5] It is well known that strained disilanes can react with dioxygen to give disiloxanes; see, for example: Atwell W. H., Uhlmann J. G. (1973) J. Organomet. Chem. 52: C21; Seyferth D., Vick 
S. C. (1977) J. Organomet. Chem. 125: C11; Sakurai H., Kobayashi T., Nakadaira Y. (1978) J. Organomet. Chem. 162: C43

[6] Krallmann R. (1991) Thesis, Bielefeld, p. 158

[7] It cannot be ruled out that ferrocenium ions participate in the reaction as catalyst: Tamao K., Kumada M., Takahashi T. (1975) J. Organomet. Chem. 94: 367

[8] Elschenbroich C., Salzer A. (1988) Organometallchemie, 2nd ed. Teubner, Stuttgart, p. 380; Freyberg D. P., Robbins J. L., Raymond K. N., Smart J. C. (1979) J. Am. Chem. Soc. 101: 892

[9] Wells A. F. (1991) Structural Inorganic Chemistry, 5th ed. (4th corr. repr.). Clarendon Press, Oxford, p. 1000

[10] There has been some controversy about the reasons for this unusual behaviour; for a comprehensive discussion, see: Shambayati S., Blake J. F., Wierschke S. G., Jorgensen W. L., Schreiber S. L. (1990) J. Am. Chem. Soc. 112: 697

[11] Additional material to the structure determination can be ordered from Fachinformationszentrum Energie-Physik-Mathematik, D-76344 Eggenstein-Leopoldshafen, Federal Republic of Germany, referring to the deposition numbers CSD-57418, to the names of the authors and the citation of the paper.

[12] Ibers J. A., Hamilton W. C. (eds.) (1974) International Tables for X-ray Crystallography, Vol. IV. Kynoch Press, Birmingham

Received June 29, 1993. Accepted July 16, 1993 